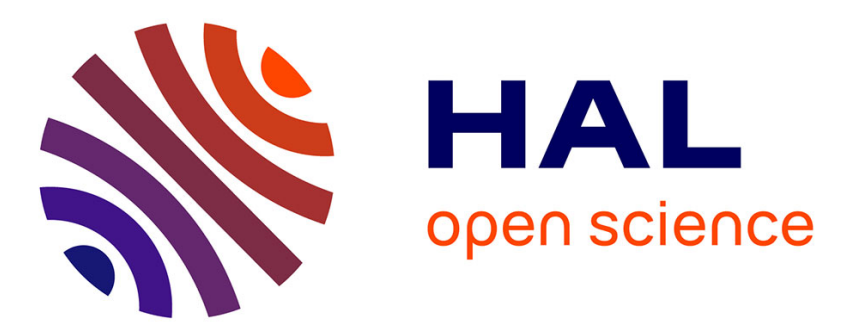

\title{
A distributed model predictive control approach for robust postural stability of a humanoid robot
}

\author{
Aurelien Ibanez, Philippe Bidaud, Vincent Padois
}

\section{To cite this version:}

Aurelien Ibanez, Philippe Bidaud, Vincent Padois. A distributed model predictive control approach for robust postural stability of a humanoid robot. IEEE International Conference on Robotics and Automation (ICRA), Jun 2014, Hong-Kong, China. pp.202 - 209, 10.1109/ICRA.2014.6906610 . hal01076570

\author{
HAL Id: hal-01076570 \\ https://hal.science/hal-01076570
}

Submitted on 22 Oct 2014

HAL is a multi-disciplinary open access archive for the deposit and dissemination of scientific research documents, whether they are published or not. The documents may come from teaching and research institutions in France or abroad, or from public or private research centers.
L'archive ouverte pluridisciplinaire HAL, est destinée au dépôt et à la diffusion de documents scientifiques de niveau recherche, publiés ou non, émanant des établissements d'enseignement et de recherche français ou étrangers, des laboratoires publics ou privés. 


\title{
A Distributed Model Predictive Control approach for robust postural stability of a humanoid robot
}

\author{
Aurelien Ibanez ${ }^{1}$, Philippe Bidaud ${ }^{1,2}$ and Vincent Padois ${ }^{1}$
}

\begin{abstract}
A novel formulation of the synthesis of motor coordination for humanoid whole-body motion is proposed in this paper, in order to ensure robust control of postural stability. It relies on the distributed model predictive control framework to coordinate, in an optimal way, several objectives. The effectiveness of this control technique to maintain postural stability of a biped against strong external disturbances is shown. Control of the horizontal dynamics of the center of mass can withstand limited perturbations. Thus postural stability criteria are specified with respect to the robot center of mass vertical and horizontal dynamics, and to the angular dynamics of its torso. Formulating the balance problem in a predictive form and distributing at different time scales significantly increases the robustness of the system to external disturbances, in terms of both tip-over and slippage risks. This original control architecture is validated through the simulation of an iCub robot performing a walking activity under unknown external actions.
\end{abstract}

\section{INTRODUCTION}

The work presented in this paper introduces a robust whole-body control approach to ensure postural stability of multi-legged locomotion systems, more particularly bipeds, against external perturbations. These perturbations, which may be of different nature (unexpected actions from the environment, uncertainty on contact conditions), affect the stability of contacts and consequently the control of the locomotion system as a whole, possibly causing the biped to tip-over or the contacts to slip. Several types of compensatory motions can be involved to improve the control of biped postural stability: horizontal [1], [2] and vertical [3] dynamics of the system, angular momentum [4], [5], actions induced by the upper-limbs [6] or recovery steps [7], [8]. The framework of Distributed Model Predictive Control (DMPC) offers to combine in an optimal way these different motor activities. The predictive aspect of such an architecture brings robustness to postural balance through the preview of future consequences of inputs on the system. The distributed aspect allows to deal with conflicting objectives, with the additional ability to adapt preview horizons to the dynamics of the controlled sub-processes.

In this paper, a control architecture for biped postural stability is developed according to this approach. Several models of the system dynamics are coordinated in order

\footnotetext{
${ }^{1}$ Aurelien Ibanez, Philippe Bidaud and Vincent Padois are with:

- Sorbonne Universités, UPMC Univ Paris 06, UMR 7222, Institut des Systèmes Intelligents et de Robotique, F-75005, Paris, France

- CNRS Centre National de la Recherche Scientifique, UMR 7222, Institut des Systèmes Intelligents et de Robotique, F-75005, Paris, France \{ibanez, bidaud, padois\}eisir.upme.fr

${ }^{2}$ Philippe Bidaud is with the ONERA, 91123 Palaiseau, France philippe.bidaudeonera.fr
}

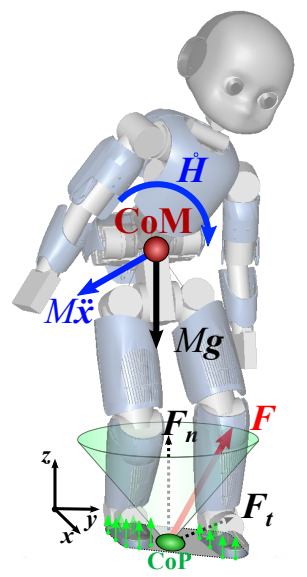

Fig. 2. Overview of postural stability dynamics

to simultaneously optimize multiple balance and contacts stability objectives, previewed over different future horizons.

The control problem of balance and stable biped locomotion for humanoid robots is often tackled through the control of some characteristic point such as the Zero-Moment Point [9] (ZMP) or Capture Point [4] (CP). The most robust applications of such criteria generally implement a predictive problem in order to provide anticipative features to the reaction of the system. A significant breakthrough in balance control involved a ZMP criterion in a predictive approach [1] and later in a Model Predictive Control (MPC) problem [2], introducing explicitly a set of constraints acting on the ZMP into the optimization problem and leading to significant improvements in robustness against strong perturbations. Robust control algorithms for walking activities were developed based on CP dynamics [10] and are subject to further developments in a MPC framework [11].

Predictive approaches are not applied to all aspects of robot control; indeed the formulation of an optimization problem over a preview horizon tends to regulate control inputs, its main advantage being the rejection of short-term disturbances. In that sense long preview horizons are not suitable to the control of systems of high-frequency dynamics. The MPC framework yet provides an indisputable gain in robustness for balance control. However, the computational cost it adds makes it more favorable to models of the system dynamics of a higher level of abstraction. A constrained Linear Inverted Pendulum (LIP) model of constant altitude is commonly used to derive the dynamics of the ZMP or CP. In addition, a significant amount of valuable developments based on the ZMP Preview Control framework [1] rely solely on the control of the horizontal dynamics of the center of mass $(\mathrm{CoM})$ of the robot to maintain balance, and rotational effects 


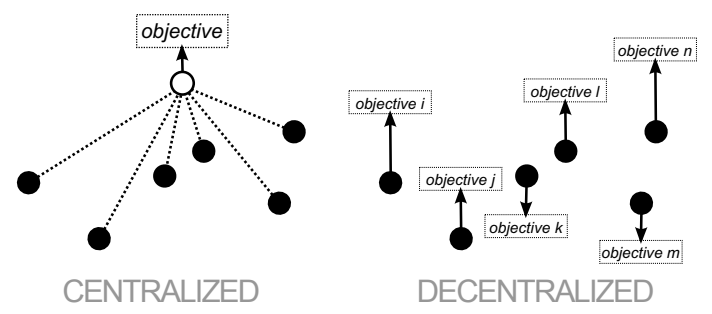

(a)

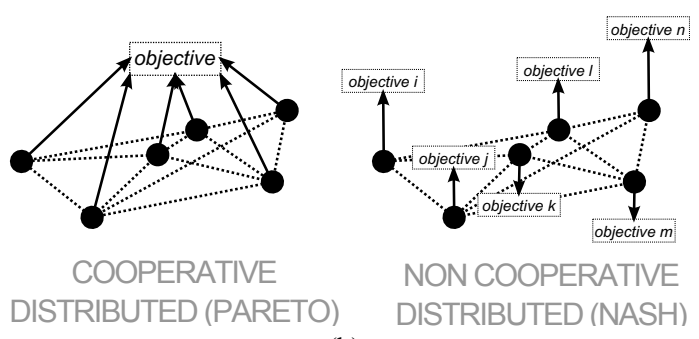

(b)

Fig. 1. Illustration of centralized, decentralized and distributed optimization problems

are commonly neglected in the ZMP equations. In order to increase balance robustness against strong perturbations, such as external pushes or ground irregularities, the introduction of additional controlled degrees of freedom is necessary; focus has been recently put in this direction towards the computation of recovery steps [12], [8], [13] to avoid the fall of the robot. These approaches hold tip-over as the main fall scenario. Contact stability is largely considered as a low-level (high priority) constraint exclusively [14], [15], [16] and no optimization is made towards the minimization of slippage risk. Nevertheless slippage is also a major concern as it leads to uncontrollable traction from the ground, and contact conditions strongly influence motor activity for example in sit-to-stand motions [17].

Contribution of this paper is directed to three aspects of predictive balance control, in an attempt to answer to the foregoing remarks. First, postural stability is maintained through both balance (tip-over) and contacts stability (slippage) maximization. Second, balance control takes advantage of both horizontal and vertical CoM dynamics, along with rotational effects through angular momentum of the robot around its CoM, as illustrated in figure 2. Last, several models of different levels of abstraction and objectives are combined within a single distributed predictive problem, and span multiple preview horizons.

The paper is organized as follows. Distribution methods are first introduced to highlight their advantages in solving large optimization problem. An optimal postural stability control problem is next developed, and its resolution is presented in the following section through decomposition and distribution at different time scales. Last section validates the resulting control architecture through the simulation of a walking activity under external pushes, and exhibits noticeable gains in robustness with respect to tip-over and slippage risks.

\section{DISTRIBUTED MODEL PREDICTIVE CONTROL}

Optimal control of systems with numerous degrees of freedom for multi-task activities generally requires the setup of a proper control architecture. A centralized architecture is the most intuitive and presents ascertained optimality properties: it consists in using a complete model of the controlled system capturing internal couplings between its different sub-processes. The monolithic structure of such an architecture raises several obstacles mainly related to computational cost that lead to a paradigm of decentralized control. Decentralized control has been subject to various developments in the last couple of decades in the field of industrial control [18], and focuses on the decomposition of the system in autonomous sub-processes as illustrated in figure 1(a).

However, in the case of strongly coupled sub-systems, adhoc techniques have to be setup in order to guarantee clear optimality properties or even stability [19]. An alternative to decentralized control is a two-layers architecture consisting in a set of autonomous sub-systems regulated by a higherlevel coordinator with respect to global objectives. In the case of conflicting subsystems, coordination consists mainly in a hierarchy [20]. Examples of coordinated architectures can be found applied to humanoid robotics with the works of [21] and [16]: the distribution of degrees of freedom among a set of local objectives (tasks) is solved either by a strict hierarchy [22] or a weighting strategy [23], respectively.

Distributed methods propose to balance drawbacks and advantages of each architecture by taking advantage from the structure of the controlled system: a communication network between sub-systems is enforced in order to reach a consensus. Communication can be bilateral (parallel distribution) or unilateral (sequential) distribution, depending on the considered coupling between sub-processes.

The Model Predictive Control (MPC) framework is notably favorable to distributed techniques as problems of large dimension are considered and it offers a time window within which a coordination strategy can be settled. Two classes of Distributed MPC (DMPC) architectures are distinguished depending on the range of the cost function considered as illustrated in table I.

\begin{tabular}{l|c|c}
\hline Objective & \multicolumn{2}{|c}{$g\left(u_{1}, u_{2}\right) \triangleq \omega_{1} g_{1}\left(u_{1}, u_{2}\right)$} \\
$+\omega_{2} g_{2}\left(u_{1}, u_{2}\right)$
\end{tabular}

TABLE I

DISTRIBUTED PROBLEMS AND RESPECTIVE OPTIMALITY

Non-cooperative distributed architectures implement subproblems with local objectives: the resulting problem is equivalent to a non-cooperative game where each sub-process only cooperates with others if it can benefit from this strategy, and convergence of such a problem is towards a Nash [24] equilibrium. A cooperative architecture considers 


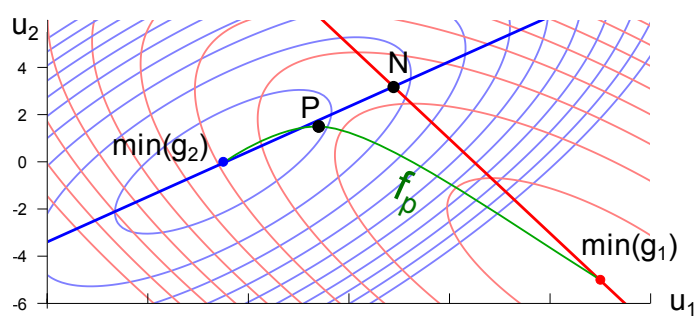

Fig. 3. Illustration of optimality from different coordination techniques: Pareto (P) and Nash (N) optima for two concurrent objectives $g_{1}\left(u_{1}, u_{2}\right)$ and $g_{2}\left(u_{1}, u_{2}\right)$ with their Pareto frontier $f_{p}$.

a shared global objective for every sub-problems and thus constitutes a team problem where all sub-processes cooperate in order to reach a minimal cost, i.e. a Pareto optimum. A comparison of these architectures is made in figure 1(b).

Note that in the case of independent sub-systems Pareto efficiency and Nash equilibrium are identical; however if sub-processes are strongly interconnected a large gap might stand between the two optima, as illustrated in figure 3. Noncooperative architectures are thus highly advantageous in computational cost terms by considering local optimization problems solely, but might not converge to a global optimum in the case of highly interdependent sub-systems. Alternatives such as dual decomposition techniques [25] allow to rewrite a team problem as a non-cooperative game, and hence set equivalence between a Nash equilibrium and a desired Pareto optimum.

The following section describes the biped stability control problem as a DMPC problem with concurrent and conflicting local objectives.

\section{CONTROL PROBLEM}

Whole-body control methods for humanoids aim at executing stable motion activities. Apart from joint-related constraints, the most severe restrictions that controllers have to cope with are related to contact points; in the case of biped walking, the stability of contacts at the feet (no slippage) and the guarantee to ensure postural balance (no tipping over) through unilateral actions are preponderant. A common way of achieving this objective is to compute the horizontal dynamics of the CoM securing postural balance - through a ZMP Preview Control [1] method, for example - to finally compute the whole-body control torques achieving this desired safe CoM behavior while respecting, among others, contact constraints [14], [15], [16].

Postural stability is ensured in this work through the minimization of both tip-over and slippage risks: contacts stability is explicitly accounted for in the preview problem along with biped balance. To achieve this objective CoM control must involve more degrees of freedom than just its horizontal dynamics: optimal future trajectories of the CoM linear acceleration and rate of angular momentum are computed online to maximize postural stability.

Scalars are noted $a$, vectors $\mathbf{a}$ and matrices $\mathbf{A} ; a_{k}$ denotes the measured variable $a$ at control step $k$ and $a_{k+i \mid k}$ the previewed value of $a$ at step $k+i$ from measured state at step $k$.

\section{A. Postural stability}

Postural stability of a humanoid robot is solely supported by contact reactions from the environment. A straightforward analysis of biped balance raises two main failure cases: tipping over and slippage. Such situations are commonly characterized by the relative amplitudes of the contact actions (friction) and their distribution on the contact areas (tip-over): their main influence in the system dynamics can hence be found respectively in the force and momentum expressions of the system equations of motion. Two postural stability criteria are derived from the whole-body dynamics expressed at the CoM $G$ of the system. These dynamics correspond in the Lagrange formalism to the equations of motion projected in the variety spanned by the unactuated degrees of freedom and are illustrated in figure 2.

Let $\mathbf{c}, z$ respectively denote the horizontal and vertical positions of the CoM of the robot. With $F_{n}$ and $F_{t}$ the respective normal and tangential norms of the resulting reaction force from the ground, the force equation yields

$$
F_{n}^{2}=M^{2}(\ddot{z}+g)^{2}, \quad F_{t}^{2}=M^{2}\|\mathbf{c}\|^{2},
$$

where $M$ is the total mass of the system and $g$ the gravity acceleration amplitude. Note that a contact situation requires $\mathbf{F}_{n} \cdot \mathbf{z}>0$, and thus the constraint

$$
\forall t \in \mathbb{R}, \quad \ddot{z}(t)>-g
$$

needs to be enforced.

The momentum equation at any point $O$ writes

$$
\mathcal{M}_{O}^{c}+\mathbf{O G} \times M \mathbf{g}=\mathbf{O G} \times M\left[\begin{array}{ll}
\ddot{\mathbf{c}}^{T} & \ddot{z}
\end{array}\right]^{T}+\dot{\mathbf{H}}_{G},
$$

where $\dot{\mathbf{H}}_{G}$ is the rate of change of angular momentum of the system around its CoM and $\mathcal{M}_{O}^{c}$ the moment of the contact forces around $O$. Angular momentum of the robot around the CoM is assumed in this paper to be supported by the upperbody solely, with the torso as its root body of which angular position is written a along the longitudinal and lateral axes. Thus its rate of change is written $\dot{H}_{G_{x}} \triangleq \ddot{a}_{x} I_{x}$ where $I_{x}$ is the upper-body inertia around the robot CoM and along the $x$ axis, with a similar expression along the $y$ axis. Assuming that all contacts are coplanar in plane $(\mathbf{x}, \mathbf{y})$, the center of pressure $(\mathrm{CoP})$ is the point on the ground of position $\mathbf{p}$ where $\mathcal{M}_{P}^{c} \cdot \mathbf{x}=\mathcal{M}_{P}^{c} \cdot \mathbf{y}=0$, and hence holds

$$
\begin{aligned}
& (g+\ddot{z})\left(p_{x}-c_{x}\right)+z \ddot{c}_{x}-\ddot{a}_{y} I_{y} / M=0 \\
& (g+\ddot{z})\left(p_{y}-c_{y}\right)+z \ddot{c}_{y}-\ddot{a}_{x} I_{x} / M=0 .
\end{aligned}
$$

A common way to maximize biped balance is to maximize the distance between the $\mathrm{CoP}$ and the edges of the sustentation convex hull. This is usually performed by setting a reference position $\mathbf{p}^{r}$ in the center of the contacts hull as a tracking objective for the ZMP $\mathbf{p}$, and hence maximizing postural balance is equivalent to minimizing a distance $h^{t}$ between $\mathbf{p}$ and $\mathbf{p}^{r}$. The minimal tip-over risk situation $\mathbf{p}=\mathbf{p}^{r}$ can be written, assuming $(g+\ddot{z}) \neq 0$, as the balance objective

$$
h^{t} \triangleq\left[\begin{array}{l}
(g+\ddot{z})\left(p_{x}^{r}-c_{x}\right)+z \ddot{c}_{x}-\ddot{a}_{y} I_{y} / M \\
(g+\ddot{z})\left(p_{y}^{r}-c_{y}\right)+z \ddot{c}_{y}-\ddot{a}_{x} I_{x} / M
\end{array}\right]^{2}=0 .
$$

A reduced model can be also deduced from (3) neglecting 
rotational effects $\ddot{a}$, which brings the biped balance simplified objective

$$
h^{b} \triangleq\left[\begin{array}{l}
(g+\ddot{z})\left(p_{x}^{r}-c_{x}\right)+z \ddot{c}_{x} \\
(g+\ddot{z})\left(p_{y}^{r}-c_{y}\right)+z \ddot{c}_{y}
\end{array}\right]^{2}=0 .
$$

A similar expression of the ZMP can be found in [3] and is strictly equivalent, under the assumption $\forall t \in \mathbb{R}, \dot{z}(t)=0$, to the widespread expression of the ZMP objective [1], [2], [8].

Slippage risk is measured in this work as a non-Euclidean distance $h^{s}$ to the Coulomb friction cone. A simple dry friction model considers two contact regimes: static friction if $F_{t}<\mu F_{n}$ and kinetic friction (slippage) for $F_{t}=\mu F_{n}$. To maximize contacts stability, slippage regimes should be avoided: tangential efforts are not controllable in such cases. The objective is hence to maximize a distance $h^{s}$ to the friction limit $F_{t}=\mu F_{n}$, written if $F_{t}<\mu F_{n}$

$$
h^{s} \triangleq-\log \left(\mu^{2} F_{n}{ }^{2}-F_{t}^{2}\right)+\log \left(\mu^{2} F_{n}{ }^{2}\right) .
$$

The distance $h^{s}$ is a logarithmic frontier to the slippage regime: in a minimal slippage risk situation $F_{t}=0 \Rightarrow h^{s}=0$, and $h^{s} \rightarrow \infty$ when $F_{t} \rightarrow \mu F_{n}^{-}$.

\section{B. Control parameters}

Trajectories of the CoM linear acceleration and rate of angular momentum are captured by the definition of the jerks $\mathbf{u} \triangleq \dddot{\mathbf{c}}, v \triangleq \dddot{z}$ and $\mathbf{w} \triangleq \dddot{\mathbf{a}}$. In discrete time of period $d t$, dynamics $\hat{\mathbf{c}} \triangleq\left[\begin{array}{ccc}\mathbf{c} & \dot{\mathbf{c}} & \ddot{\mathbf{c}}\end{array}\right]^{T}, \hat{\mathbf{z}}$ and $\hat{\mathbf{a}}$ can be deduced from a simple integration scheme

$$
\forall i \in \mathbb{N}, \begin{aligned}
\hat{\mathbf{c}}_{k+i+1 \mid k} & =\mathbf{A} \hat{\mathbf{c}}_{k+i \mid k}+\mathbf{B} \mathbf{u}_{k+i \mid k} \\
\hat{\mathbf{z}}_{k+i+1 \mid k} & =\mathbf{A}_{\mathbf{z}} \hat{\mathbf{z}}_{k+i \mid k}+\mathbf{B}_{\mathbf{z}} v_{k+i \mid k} \\
\hat{\mathbf{a}}_{k+i+1 \mid k} & =\mathbf{A} \hat{\mathbf{a}}_{k+i \mid k}+\mathbf{B} \mathbf{w}_{k+i \mid k} .
\end{aligned}
$$

Thus any function of $\hat{\mathbf{c}}, \hat{\mathbf{z}}$ or $\hat{\mathbf{a}}$ is written as a function of $\mathbf{u}$, $v$ or $\mathbf{w}$, respectively.

\section{Optimization}

The control problem solved in this paper aims at maximizing postural stability over a preview horizon; that is, maximize biped balance and contacts stability. Cost functions $h^{t}, h^{b}$ and $h^{s}$ involve different dynamics (vertical, horizontal and rotational) and capture these objectives at different levels: $h^{t}$ measures a tip-over risk with a view to vertical, horizontal and rotational dynamics of the system, $h^{b}$ relies on a simpler model of the system to quantify a balance error to a reference CoP position and $h^{s}$ defines a contacts stability criterion as a slippage risk with respect to vertical and horizontal dynamics. This difference in insight provided by each cost function leads to the exploitation of different preview horizons $n_{t}, n_{b}$ and $n_{s}$ respectively. In discrete time of period $d t$, the postural stability maximization problem hence writes

$$
\min \underbrace{\omega_{t} \sum_{i=1}^{n_{t}} h_{k+i \mid k}^{t}}_{H_{n_{t}}^{t}}+\underbrace{\omega_{b} \sum_{i=1}^{n_{b}} h_{k+i \mid k}^{b}}_{H_{n_{b}}^{b}}+\underbrace{\omega_{s} \sum_{i=1}^{n_{s}} h_{k+i \mid k}^{s}}_{H_{n_{s}}^{s}},
$$

where $\omega_{t}, \omega_{b}$ and $\omega_{s}$ are scalar coefficients defining the relative predominance of each individual objective and accounting for differences in dimensions. Horizons $\boldsymbol{U}, \boldsymbol{V}$ and $\boldsymbol{W}$ of control inputs $\mathbf{u}, v$ and $\mathbf{w}$ described in the previous paragraph are denoted at control step $k$

$$
\begin{aligned}
\boldsymbol{U}_{k}^{m} & \triangleq\left[\begin{array}{lll}
\mathbf{u}_{k \mid k} & \ldots & \mathbf{u}_{k+m-1 \mid k}
\end{array}\right]^{T}, \\
\boldsymbol{V}_{k}^{m} & \triangleq\left[\begin{array}{lll}
v_{k \mid k} & \ldots & v_{k+m-1 \mid k}
\end{array}\right]^{T}, \\
\boldsymbol{W}_{k}^{m} & \triangleq\left[\begin{array}{lll}
\mathbf{w}_{k \mid k} & \ldots & \mathbf{w}_{k+m-1 \mid k}
\end{array}\right]^{T} .
\end{aligned}
$$

Let $N \triangleq \max \left(n_{t}, n_{b}, n_{s}\right)$; the optimization problem derived from the postural stability cost (7) writes

$$
\left\{\begin{aligned}
\min _{\boldsymbol{U}_{k}^{N}, \boldsymbol{V}_{k}^{N}, \boldsymbol{W}_{k}^{n_{t}}} & H_{n_{t}}^{t}\left(\boldsymbol{U}_{k}^{n_{t}}, \boldsymbol{V}_{k}^{n_{t}}, \boldsymbol{W}_{k}^{n_{t}}\right) \\
& +H_{n_{b}}^{b}\left(\boldsymbol{U}_{k}^{n_{b}}, \boldsymbol{V}_{k}^{n_{b}}\right)+H_{n_{s}}^{s}\left(\boldsymbol{U}_{k}^{n_{s}}, \boldsymbol{V}_{k}^{n_{s}}\right) \\
\text { s.t. } & \quad(2) .
\end{aligned}\right.
$$

Note that no straightforward convexity properties can be found in $H^{t}, H^{b}$ and $H^{s}$. However an admissible initial guess can be derived from an unconstrained implementation of a ZMP Preview controller [1], [2] for example and a local optimum within its neighborhood can be computed. The optimization problem is regularized with the introduction of additional strictly convex objective functions for each control parameter. A generalized Tikhonov regularization method is introduced with the following additional convex objective functions

$$
\begin{aligned}
& H_{n}^{u} \triangleq \omega_{u} \sum_{i=0}^{n-1}\left\|\mathbf{u}_{k+i \mid k}\right\|^{2}, H_{n}^{z} \triangleq \sum_{i=1}^{n}\left\|\boldsymbol{\Omega}_{z}\left(\hat{\mathbf{z}}_{k+i \mid k}-\hat{\mathbf{z}}^{r}\right)\right\|^{2}, \\
& H_{n}^{v} \triangleq \omega_{v} \sum_{i=0}^{n-1} v_{k+i \mid k}^{2} \text { and } H_{n}^{w} \triangleq \sum_{i=1}^{n}\left\|\boldsymbol{\Omega}_{w}\left(\hat{\mathbf{a}}_{k+i \mid k}-\hat{\mathbf{a}}^{r}\right)\right\|^{2},
\end{aligned}
$$

where $\omega_{u}$ and $\omega_{v}$ are scalar coefficients, $\boldsymbol{\Omega}_{z}$ and $\boldsymbol{\Omega}_{w}$ diagonal weighting matrices. $H^{u}$ and $H^{v}$ can be interpreted as objective terms giving preference to smaller norm solutions. Additional costs $H^{z}$ and $H^{w}$ describe supplementary tasks maintaining the $\mathrm{CoM}$ vertical position and torso orientation around reference states; note that desired reference states $\hat{\mathbf{z}}^{r}$ and $\hat{\mathbf{a}}^{r}$ are not necessarily constant and can be used to add a desired trajectory as an additional objective.

The postural stability control problem is written as the following regularized optimization problem

$$
\left\{\begin{aligned}
\min _{\boldsymbol{U}_{k}^{N}, \boldsymbol{V}_{k}^{N}, \boldsymbol{W}_{k}^{n_{t}}} & G_{n_{t}}^{t}\left(\boldsymbol{U}_{k}^{n_{t}}, \boldsymbol{V}_{k}^{n_{t}}, \boldsymbol{W}_{k}^{n_{t}}\right) \\
& +G_{n_{b}}^{b}\left(\boldsymbol{U}_{k}^{n_{b}}, \boldsymbol{V}_{k}^{n_{b}}\right)+G_{n_{s}}^{s}\left(\boldsymbol{U}_{k}^{n_{s}}, \boldsymbol{V}_{k}^{n_{s}}\right) \\
\text { s.t. } \quad & (2),
\end{aligned}\right.
$$

where

$$
\begin{aligned}
G_{n_{t}}^{t}\left(\boldsymbol{U}_{k}^{n_{t}}, \boldsymbol{V}_{k}^{n_{t}}, \boldsymbol{W}_{k}^{n_{t}}\right) \triangleq & H_{n_{t}}^{t}\left(\boldsymbol{U}_{k}^{n_{t}}, \boldsymbol{V}_{k}^{n_{t}}, \boldsymbol{W}_{k}^{n_{t}}\right) \\
& +H_{n_{t}}^{w}\left(\boldsymbol{W}_{k}^{n_{t}}\right), \\
G_{n_{b}}^{b}\left(\boldsymbol{U}_{k}^{n_{b}}, \boldsymbol{V}_{k}^{n_{b}}\right) \triangleq & H_{n_{b}}^{b}\left(\boldsymbol{U}_{k}^{n_{b}}, \boldsymbol{V}_{k}^{n_{b}}\right) \\
& +H_{n_{b}}^{u}\left(\boldsymbol{U}_{k}^{n_{b}}\right)+H_{n_{b}}^{z}\left(\boldsymbol{V}_{k}^{n_{b}}\right), \\
\text { and } G_{n_{s}}^{s}\left(\boldsymbol{U}_{k}^{n_{s}}, \boldsymbol{V}_{k}^{n_{s}}\right) \triangleq & H_{n_{s}}^{s}\left(\boldsymbol{U}_{k}^{n_{s}}, \boldsymbol{V}_{k}^{n_{s}}\right) \\
& +H_{n_{s}}^{v}\left(\boldsymbol{V}_{k}^{n_{s}}\right)
\end{aligned}
$$

are the three regularized cost functions of problem (8).

The following section presents the distribution method proposed to solve such an optimization problem. 


\section{RESOLUTION}

Concurrency between balance models is first distributed and conflicts between stability objectives are next exposed and relaxed through dual decomposition. The resulting DMPC problem is solved with an implementation of a parallelized projected gradient algorithm.

\section{A. Sequential distribution}

A sequential distribution is performed to uncouple the rotational variable $\boldsymbol{W}_{k}$ from the linear ones. Problem (9) can be divided into two optimization problems, with $N \triangleq \max \left(n_{b}, n_{s}\right)$

$$
\left\{\begin{array}{c}
\min _{\boldsymbol{U}_{k}^{N}, \boldsymbol{V}_{k}^{N}} G_{n_{b}}^{b}\left(\boldsymbol{U}_{k}^{n_{b}}, \boldsymbol{V}_{k}^{n_{b}}\right)+G_{n_{s}}^{s}\left(\boldsymbol{U}_{k}^{n_{s}}, \boldsymbol{V}_{k}^{n_{s}}\right) \\
\text { s.t. (2), } \\
\min _{\boldsymbol{U}_{k}^{n_{t}}, \boldsymbol{V}_{k}^{n_{t}}, \boldsymbol{W}_{k}^{n_{t}}} G_{n_{t}}^{t}\left(\boldsymbol{U}_{k}^{n_{t}}, \boldsymbol{V}_{k}^{n_{t}}, \boldsymbol{W}_{k}^{n_{t}}\right) \\
\text { s.t. (2) }
\end{array}\right.
$$

which are coupled by the variables $\boldsymbol{U}_{k}$ and $\boldsymbol{V}_{k}$. The distribution adopted consists in solving the problem (10b) after (10a): $\boldsymbol{U}_{k}$ and $\boldsymbol{V}_{k}$ are considered as inputs to the problem (10b). The centralized problem (9) is hence distributed as follows

$$
\begin{aligned}
& \text { (Find } \boldsymbol{U}_{k}^{N *}, \boldsymbol{V}_{k}^{N *} \text { that solve } \\
& \min _{\boldsymbol{U}_{k}^{N}, \boldsymbol{V}_{k}^{N}} G_{n_{b}}^{b}\left(\boldsymbol{U}_{k}^{n_{b}}, \boldsymbol{V}_{k}^{n_{b}}\right)+G_{n_{s}}^{s}\left(\boldsymbol{U}_{k}^{n_{s}}, \boldsymbol{V}_{k}^{n_{s}}\right) \\
& \text { s.t. (2), } \\
& \text { Find } \boldsymbol{W}_{k}^{n_{t *}} \text { that solves } \\
& \begin{array}{l}
\min _{\boldsymbol{W}_{k}^{n_{t}}} G_{n_{t}}^{t}\left(\boldsymbol{U}_{k}^{n_{t *}}, \boldsymbol{V}_{k}^{n_{t *}}, \boldsymbol{W}_{k}^{n_{t}}\right) \\
\text { s.t. }
\end{array}
\end{aligned}
$$

which is not strictly equivalent. Nevertheless such a distribution can be interpreted as the consideration of angular momentum as a non-conflicting means to increase the balance performance: optimal CoM linear dynamics are first computed unaware of rotational effects through (11a), and the torso angular dynamics are subsequently adjusted according to (11b) in order to maximize the postural stability of the system. This sequential distribution (11) of (9) is adopted in this work to reduce the influence of $H^{t}$ over $\boldsymbol{U}_{k}$ and $\boldsymbol{V}_{k}$ to a unilateral pairing, due to the error-prone approximation made on the angular momentum support.

\section{B. Dual decomposition}

The coupling between balance and stability objectives $G^{b}$ and $G^{s}$ of problem (11a) cannot be handled safely through a straightforward distribution as introduced in the previous paragraph. Their concurrency indeed induces that any change in $\boldsymbol{U}_{k}$ or $\boldsymbol{V}_{k}$ that would benefit $G^{b}$ might deteriorate the distance $G^{s}$ : such problems can be referred to as cooperative - or team - problems. Dual decomposition allows to set up a market mechanism where $G^{b}$ and $G^{s}$ are locally optimized while both problems are driven to a consensus. The resulting strictly equivalent problem is of a simpler structure - non-cooperative - and thus more favorable to common solving algorithms.
Problem (11a) can be written as the following constrained problem by the introduction of local versions $(\boldsymbol{\Phi}, \boldsymbol{\Theta})$ of the coupling variables $(\boldsymbol{U}, \boldsymbol{V})$

$$
\left\{\begin{aligned}
\min _{\substack{\boldsymbol{U}_{k}^{N}, \boldsymbol{V}_{k}^{N}, \boldsymbol{\Phi}_{k}^{N}, \boldsymbol{\Theta}_{k}^{N} \\
\text { s.t. }}} G_{n_{b}}^{b}\left(\boldsymbol{U}_{k}^{n_{b}}, \boldsymbol{\Theta}_{k}^{n_{b}}\right)+G_{n_{s}}^{s}\left(\boldsymbol{\Phi}_{k}^{n_{s}}, \boldsymbol{V}_{k}^{n_{s}}\right) \\
\text { s. }\left\{\begin{array}{l}
(2), \\
\boldsymbol{\Phi}_{k}^{n}-\boldsymbol{U}_{k}^{n}=\mathbf{0}, \\
\boldsymbol{\Theta}_{k}^{n}-\boldsymbol{V}_{k}^{n}=\mathbf{0}
\end{array}\right.
\end{aligned}\right.
$$

where $n \triangleq \min \left(n_{b}, n_{s}\right)$. The Lagrangian $\mathcal{L}$ of this problem writes, with the Lagrange multipliers $\boldsymbol{\theta}$ and $\phi$

$$
\begin{aligned}
\mathcal{L} \triangleq & G_{n_{b}}^{b}\left(\boldsymbol{U}_{k}^{n_{b}}, \boldsymbol{\Theta}_{k}^{n_{b}}\right)+G_{n_{s}}^{s}\left(\boldsymbol{\Phi}_{k}^{n_{s}}, \boldsymbol{V}_{k}^{n_{s}}\right) \\
& +\boldsymbol{\phi}_{k}^{n T}\left[\boldsymbol{\Phi}_{k}^{n}-\boldsymbol{U}_{k}^{n}\right]+\boldsymbol{\theta}_{k}^{n T}\left[\boldsymbol{\Theta}_{k}^{n}-\boldsymbol{V}_{k}^{n}\right]
\end{aligned}
$$

and problem (12) - problem (11a) consequently - is strictly equivalent to

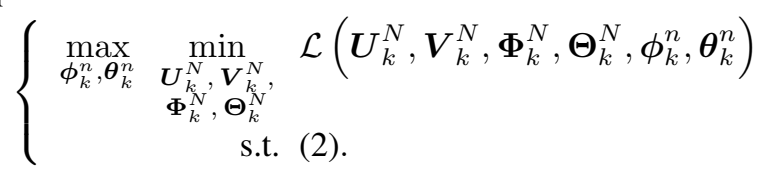

This problem is separable, and can be dissociated into four subproblems

$$
\left\{\begin{array}{l}
\min _{\boldsymbol{U}_{k}^{N}, \boldsymbol{\Theta}_{k}^{n_{b}}} G_{n_{b}}^{b}\left(\boldsymbol{U}_{k}^{n_{b}}, \boldsymbol{\Theta}_{k}^{n_{b}}\right)+\boldsymbol{\theta}_{k}^{n T} \boldsymbol{\Theta}_{k}^{n}-\boldsymbol{\phi}_{k}^{n T} \boldsymbol{U}_{k}^{n} \\
\min _{\boldsymbol{\Phi}_{k}^{n_{s}}, \boldsymbol{V}_{k}^{N}} G_{n_{s}}^{s}\left(\boldsymbol{\Phi}_{k}^{n_{s}}, \boldsymbol{V}_{k}^{n_{s}}\right)+\boldsymbol{\phi}_{k}^{n T} \boldsymbol{\Phi}_{k}^{n}-\boldsymbol{\theta}_{k}^{n T} \boldsymbol{V}_{k}^{n} \\
\max _{\boldsymbol{\phi}_{k}^{n}} \boldsymbol{\phi}_{k}^{n T}\left[\boldsymbol{\Phi}_{k}^{n}-\boldsymbol{U}_{k}^{n}\right] \\
\max _{\boldsymbol{\theta}_{k}^{n}} \boldsymbol{\theta}_{k}^{n T}\left[\boldsymbol{\Theta}_{k}^{n}-\boldsymbol{V}_{k}^{n}\right] \\
\text { s.t. (2). }
\end{array}\right.
$$

The set of problems (13) can be interpreted as a market mechanism where $\boldsymbol{\theta}$ and $\phi$ regulate the cost of violating the coupling between the objectives $G^{b}$ and $G^{s}$. Such a distributed architecture represents a non-cooperative parallel problem: each subproblem optimizes a local cost function with its own variables. Communication between them is sufficient to reach a Nash equilibrium of problem (13) which is strictly equivalent to the desired Pareto optimum of the centralized problem (11a).

\section{Algorithm}

Although problems (13c) and (13d) are always convex, problems (13a) and (13b) present no peculiar convexity properties; however an admissible local optimum around an initial guess is considered as satisfactory in this work. The linear constraint (2) and the previous remarks license the setup of a projected gradient algorithm to solve problem (13). Note that this algorithm is naturally decomposed in four processes.

Problem (13) is written for the sake of simplicity

$$
\left\{\begin{array}{l}
\min _{\boldsymbol{U}_{k}^{N}, \boldsymbol{\Theta}_{k}^{n_{b}}} J^{b}\left(\boldsymbol{U}_{k}^{n_{b}}, \boldsymbol{\Theta}_{k}^{n_{b}}, \boldsymbol{\theta}_{k}^{n}, \boldsymbol{\Theta}_{k}^{n}, \boldsymbol{\phi}_{k}^{n}\right), \text { s.t. (2) } \\
\min _{\boldsymbol{\Phi}_{k}^{n_{s}}, \boldsymbol{V}_{k}^{N}} J^{s}\left(\boldsymbol{\Phi}_{k}^{n_{s}}, \boldsymbol{V}_{k}^{n_{s}}, \boldsymbol{\phi}_{k}^{n}, \boldsymbol{\Phi}_{k}^{n}, \boldsymbol{\theta}_{k}^{n}\right), \text { s.t. (2) } \\
\\
\min _{\boldsymbol{\phi}_{k}^{n}} J^{\phi}\left(\boldsymbol{\phi}_{k}^{n}, \boldsymbol{\Phi}_{k}^{n}, \boldsymbol{U}_{k}^{n}\right) \\
\min _{\boldsymbol{\theta}_{k}^{n}} J^{\theta}\left(\boldsymbol{\theta}_{k}^{n}, \boldsymbol{\Theta}_{k}^{n}, \boldsymbol{V}_{k}^{n}\right)
\end{array}\right.
$$


where

$$
\begin{aligned}
J^{\phi}\left(\boldsymbol{\phi}_{k}^{n}, \boldsymbol{\Phi}_{k}^{n}, \boldsymbol{U}_{k}^{n}\right) & =\boldsymbol{\phi}_{k}^{n T}\left[\boldsymbol{U}_{k}^{n}-\boldsymbol{\Phi}_{k}^{n}\right], \\
J^{\theta}\left(\boldsymbol{\theta}_{k}^{n}, \boldsymbol{\Theta}_{k}^{n}, \boldsymbol{V}_{k}^{n}\right) & =\boldsymbol{\theta}_{k}^{n T}\left[\boldsymbol{V}_{k}^{n}-\boldsymbol{\Theta}_{k}^{n}\right],
\end{aligned}
$$

and is solved with the parallelized algorithm described in figure 4, with $\Pi_{g}^{m}: \mathbb{R}^{m} \rightarrow \mathbb{R}^{m}$ a projector on the variety delimited by (2) over $m$ preview steps. Descent steps $\alpha$ are determined using a backtracking line search method.

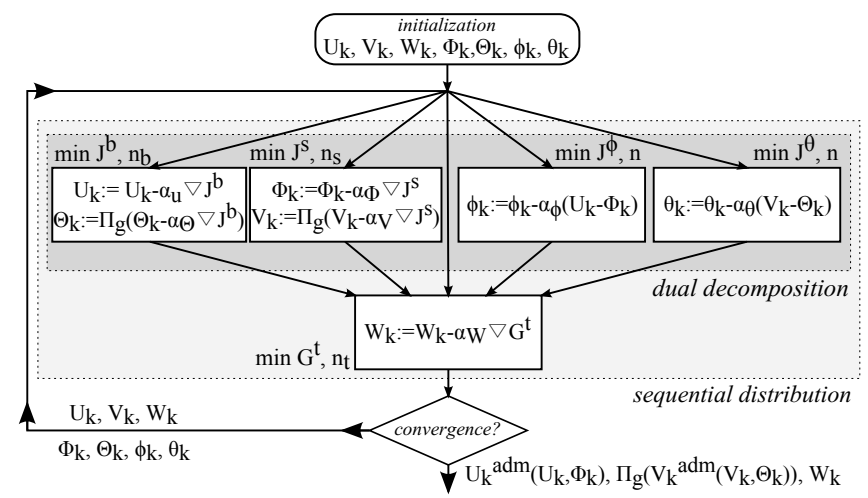

Fig. 4. Flow chart of the iterative gradient descent algorithm solving the non-cooperative and sequential distributed problems (14) and (11)

Typical convergence results for objective functions $J^{b}$ and $J^{s}$ with this algorithm are shown in figure 5: concurrency is clearly visible as both objectives successively and alternately compromise until they reach a mutual agreement. The gap between decoupled variables $(\boldsymbol{U}, \boldsymbol{\Phi})$ and $(\boldsymbol{V}, \boldsymbol{\Theta})$

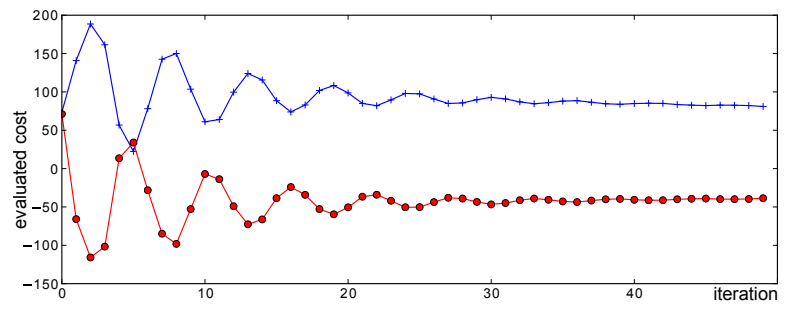

Fig. 5. Typical convergence results for objective functions $J^{b}$ and $J^{s}$ - blue cross: $J^{b}$ - red circle: $J^{s}$

is simultaneously reduced and converges towards a consensus for the values of $(\boldsymbol{U}, \boldsymbol{V})$, as shown in figure 6 . This figure shows that convergence of the pair $(\boldsymbol{V}, \boldsymbol{\Theta})$ is slower than $(\boldsymbol{U}, \boldsymbol{\Phi})$; this can be interpreted as vertical dynamics being the preponderant source of conflict between balance and friction objectives. Note that a suboptimal so-

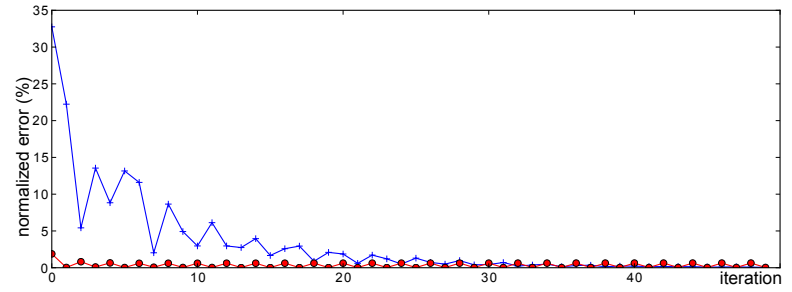

Fig. 6. Typical convergence of constraint relaxations $\boldsymbol{U}=\boldsymbol{\Phi}$ and $\boldsymbol{V}=\boldsymbol{\Theta}$ towards a consensus - blue cross: $\frac{\left\|\Theta_{k}^{n}-\boldsymbol{V}_{k}^{n}\right\|}{\left\|\boldsymbol{V}_{k}^{n}\right\|}-$ red circle: $\frac{\left\|\boldsymbol{\Phi}_{k}^{n}-\boldsymbol{U}_{k}^{n}\right\|}{\left\|\boldsymbol{U}_{k}^{n}\right\|}$

lution can be obtained by stopping the algorithm before convergence is reached: an admissible solution with respects to the coupling constraints is obtained by projections $\boldsymbol{U}_{k}^{n a d m}=\frac{\boldsymbol{U}_{k}^{n}+\boldsymbol{\Phi}_{k}^{n}}{2}$ and $\boldsymbol{V}_{k}^{n a d m}=\frac{\boldsymbol{V}_{k}^{n}+\boldsymbol{\Theta}_{k}^{n}}{2}$ onto the feasible sets $\boldsymbol{U}=\boldsymbol{\Phi}$ and $\boldsymbol{V}=\boldsymbol{\Theta}$. Note that convergence results in figures 5 and 6 are obtained from the simulations presented in Sec. V: less than 30 descent iterations were required to reach convergence.

\section{RESULTS}

Simulations have been carried out using ArborisPython [26], an open-source dynamics simulator developed at ISIR with the Python programming language. An accurate model of an iCub robot [27] is simulated with $32+6$ degrees of freedom.

\section{A. Compared frameworks}

Whole-body control of the model is ensured by an LQPbased controller [16] accounting for joint and contact constraints. Contacts between the robot and the ground are solved according to a dry friction model of static coefficient $\mu=0.5$. The whole-body controller takes as inputs desired trajectories of the CoM position and torso orientation, and the performance of the DMPC algorithm introduced in this work is compared to the one of a state of the art predictive framework.

A low-priority task is defined for both frameworks to try and maintain all joints position around a predefined reference describing a standing pose of the robot. Trajectory tracking tasks for both the CoM position and the torso orientation are included in the whole-body control problem as top-level priority tasks (weight a hundred times greater in magnitude). The predictive framework is centered around an unconstrained implementation of the ZMP Preview Control as described in [2] which provides optimal horizontal trajectories for the CoM of the robot. Orientation of the torso is indirectly maintained by the low priority reference task, while CoM altitude is regulated with a stiff proportionalderivative controller in order to enforce the constant-altitude assumption made in the ZMP Preview Control formulation [1].

In the second framework all CoM and torso desired trajectories are output from the DMPC algorithm introduced in this paper, as shown in figure 7.
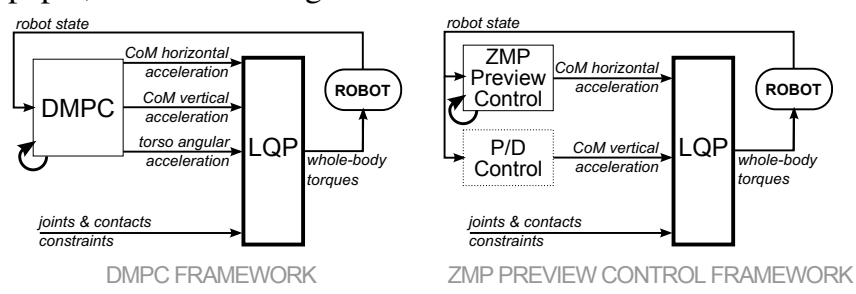

Fig. 7. Predictive control frameworks compared

\section{B. Simulation results}

The scenario supporting the simulation is a walking activity under an unknown external action from the environment on the waist of the robot, as illustrated with a selection of simulation snapshots in figure 8 .

The profile of the external action is shown in figure 9 which exhibits two impulses in the horizontal plane shortly preceding the changes of supporting foot. 


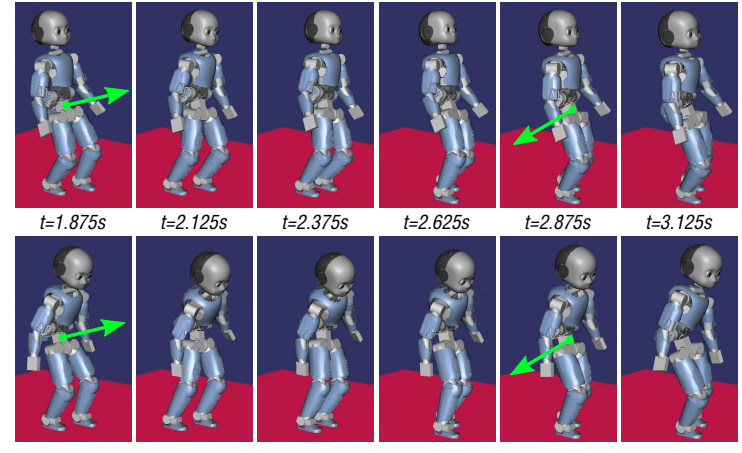

Fig. 8. Simulation snapshots for both control frameworks - top: ZMP Preview control - bottom: DMPC

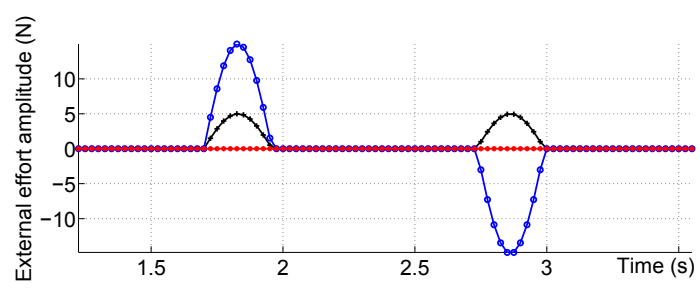

Fig. 9. Amplitude profile of the unknown external action applied on the waist of the robot - blue circle: lateral - black cross: longitudinal - red star: vertical

As the cost function $G^{b}$ is the closest in insight - among $G^{b}, G^{t}$ and $G^{s}$ - to the objective function minimized in the ZMP Preview Control framework, its horizon $n_{b}$ is set similarly to the ZMP Preview control problem and covers $1.0 \mathrm{~s}$. The selected values for preview horizons $n_{t}$ and $n_{s}$ both span $0.25 \mathrm{~s}$. Different values of $n_{t}$ and $n_{s}$ from $n_{b} / 8$ to $n_{b}$ resulted in a lower performance of the controller during critical phases. This remark validates the need for different preview horizons depending on the level of insight provided by each control objective and the predominant dynamics it involves. To allow for comparison regularization of $H^{b}$ is set up to the same order of magnitude as in the ZMP Preview Control problem, that is $\omega_{u} / \omega_{b}=1 . e^{-6}$.

Figures 10 and 11 show the evolution of the CoP position on the ground for both frameworks, with regard to the tracked reference position. These figures show that, as

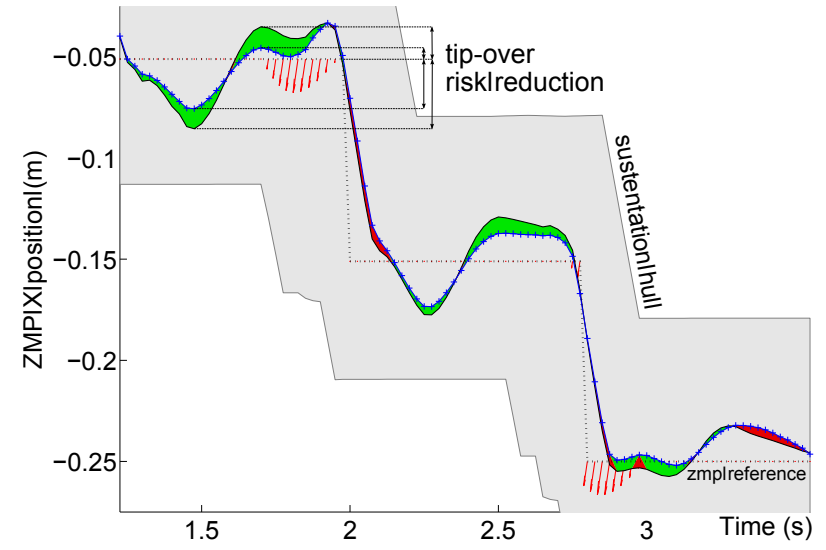

Fig. 10. Evolution of the ZMP in longitudinal direction - blue cross: DMPC - black solid: ZMP Preview Control - red arrows: normalized external effort expected, the concomitant use of both horizontal and vertical

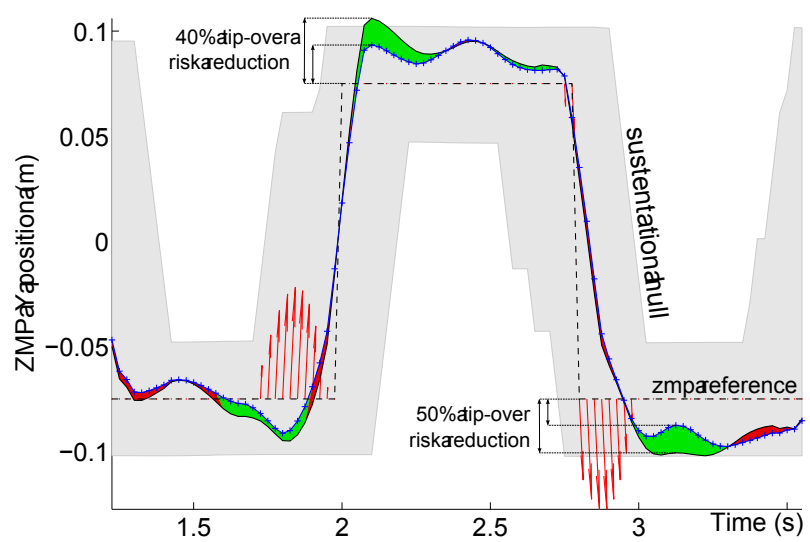

Fig. 11. Evolution of the ZMP in lateral direction - blue cross: DMPC - black solid: ZMP Preview Control - red arrows: normalized external effort

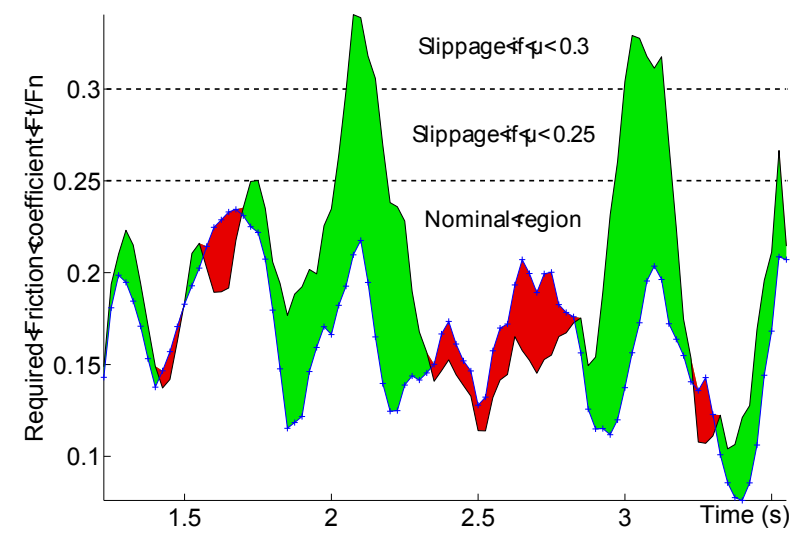

Fig. 12. Evolution of the required friction coefficient - blue cross: DMPC - black solid: ZMP Preview Control

degrees of freedom of the CoM along with the control of angular momentum allows for a significant gain in balance performance. This improvement in reference tracking can reach up to more than $50 \%$ during critical intervals, as shown at instants $t \approx 2.0 \mathrm{~s}$ and $t \approx 3.0 \mathrm{~s}$ when the ZMP Preview Control framework almost fails at maintaining the robot postural balance.

An additional benefit from the introduction of the vertical control of the CoM is displayed in figure 12 which maps the amplitude ratio $\nu$ between tangential and normal components of the resulting contact force with the ground. This ratio can be interpreted as a required friction coefficient: if the actual friction coefficient $\mu$, characterizing the contact conditions with the ground, is lower than or equal to $\nu$, then slippage occurs and might lead to postural instability. In that sense, the lowest required friction coefficient minimizes the risk of slippage in case of ill-estimation of the current contact conditions. Actual friction coefficient $\mu$ might be overestimated in a variety of usual cases in unstructured environments: strong drops in admissible traction can be observed for example if the robot steps over a wet area or an unexpected region of different material. Figure 12 shows that the robot can control the reaction forces from the ground until a limit of $\mu_{\min }=0.25$ with the DMPC framework and down to $\mu_{\min }=0.34$ with the ZMP Preview Control framework. Relatively to the expected friction coefficient $\mu=0.5$ these figures bring an uncertainty tolerance - robustness - of $50 \%$ for the DMPC 


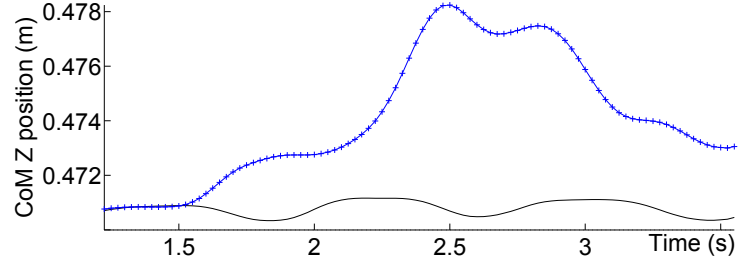

Fig. 13. Evolution of the CoM altitude - blue cross: DMPC - black solid: ZMP Preview Control

framework and less than $35 \%$ for the ZMP Preview Control framework. The consequences of this higher robustness are illustrated in the video attached to this paper, presenting a disturbed scenario where postural stability is maintained with the DMPC controller while the ZMP Preview Control framework fails at ensuring contacts stability. The DMPC framework is thus prominently more robust to disturbances than the ZMP Preview Control framework. Note that due to the exponential growth of the slippage cost function $H^{s}$, no noticeable performance gain can be found in the nominal region and only slippage-prone peaks are cut down.

Finally, the DMPC framework allows to automatically regulate the altitude of the CoM of the robot and its variations, as shown in figure 13. The ZMP Preview Control framework bounds the CoM to stay at a constant altitude in order to preserve the validity of the cart-table model, while the DMPC framework takes benefit from this degree of freedom in order to compromise between balance and stability objectives.

\section{CONCLUSIONS}

The control problem of postural stability of a humanoid robot is approached in this work in a distributed model predictive control framework. This novel formulation takes advantage of different models of the balance dynamics, coordinated along multiple future horizons, and efficiently increases the robustness of the system against strong and unexpected external perturbations.

Contributions of this control architecture are highlighted with the simulation of a walking activity under external pushes: significant gains in balance and contacts stability are observed compared to a ZMP Preview Control framework. Tip-over and slippage risks are noticeably minimized with the predictive control of the center of mass horizontal and vertical dynamics, and of the robot angular momentum.

Future works will investigate the generalization of the DMPC framework for the control problem of multi-task activities.

\section{ACKNOWLEDGMENT}

This work was partially supported by the French Ministry of Higher Education and Research, by the RTE company through its chair "Robotics Systems for field intervention in constrained environments" hold by Vincent Padois and by the European Commission within the CoDyCo project (FP7ICT-2011-9, No. 600716).

\section{REFERENCES}

[1] S. Kajita, F. Kanehiro, K. Kaneko, K. Kajiwara, K. Harada, K. Yokoi, and H. Hirukawa, "Biped walking pattern generation by using preview control of zero-moment point," in Proc. of the IEEE ICRA, 2003.
[2] P.-B. Wieber, "Trajectory free linear model predictive control for stable walking in the presence of strong perturbations," in Proc. of the IEEERAS Int. Conf. on Humanoid Rob., 2006, pp. 137-142.

[3] J. H. Park and H. C. Cho, "An online trajectory modifier for the base link of biped robots to enhance locomotion stability," in Proc. of the IEEE ICRA, vol. 4. IEEE, 2000, pp. 3353-3358.

[4] J. Pratt, J. Carff, S. Drakunov, and A. Goswami, "Capture point: A step toward humanoid push recovery," in Proc. of the IEEE-RAS Int Conf. on Humanoid Rob. IEEE, 2006, pp. 200-207.

[5] D. N. Nenchev and A. Nishio, "Ankle and hip strategies for balance recovery of a biped subjected to an impact," Robotica, vol. 26, no. 05 , pp. 643-653, 2008.

[6] A. Ibanez, P. Bidaud, and V. Padois, "Unified preview control for humanoid postural stability and upper-limb interaction adaptation," in Proc. of the IEEE/RSJ IROS. IEEE, 2012, pp. 1801-1808.

[7] B. Stephens, "Humanoid push recovery," in Proc. of the IEEE-RAS Int. Conf. on Humanoid Rob. IEEE, 2007, pp. 589-595.

[8] A. Herdt, H. Diedam, P.-B. Wieber, D. Dimitrov, K. Mombaur, and M. Diehl, "Online walking motion generation with automatic footstep placement," in $A d v$. Rob., vol. 24, no. 5-6, 2010, pp. 719-737.

[9] M. Vukobratović and J. Stepanenko, "On the stability of anthropomorphic systems," in Math. Biosciences, vol. 15, no. 1, 1972, pp. 1-37.

[10] J. Englsberger, C. Ott, M. A. Roa, A. Albu-Schaffer, and G. Hirzinger, "Bipedal walking control based on capture point dynamics," in Proc. of the IEEE/RSJ IROS. IEEE, 2011, pp. 4420-4427.

[11] M. Krause, J. Englsberger, P.-B. Wieber, and C. Ott, "Stabilization of the capture point dynamics for bipedal walking based on model predictive control," in Robot Control, vol. 10, 2012, pp. 165-171.

[12] O. Stasse, P. Evrard, N. Perrin, N. Mansard, and A. Kheddar, "Fast foot prints re-planning and motion generation during walking in physical human-humanoid interaction," in Proc. of the IEEE-RAS Int. Conf. on Humanoid Rob., 2009, pp. 284-289.

[13] B. J. Stephens and C. G. Atkeson, "Push recovery by stepping for humanoid robots with force controlled joints," in Proc. of the IEEERAS Int. Conf. on Humanoid Rob. IEEE, 2010, pp. 52-59.

[14] L. Sentis, "Synthesis and control of whole-body behaviors in humanoid systems," Ph.D. dissertation, Stanford university, 2007.

[15] N. Mansard, O. Khatib, and A. Kheddar, "A unified approach to integrate unilateral constraints in the stack of tasks," IEEE Trans. on Rob., vol. 25, no. 3, pp. 670-685, 2009.

[16] J. Salini, V. Padois, and P. Bidaud, "Synthesis of complex humanoid whole-body behavior: a focus on sequencing and tasks transitions," in Proc. of the IEEE ICRA. IEEE, 2011, pp. 1283-1290.

[17] S. Barthélemy and P. Bidaud, "Stability measure of postural dynamic equilibrium based on residual radius," in Adv. in Robot Kinematics: Analysis and Design. Springer, 2008, pp. 399-407.

[18] D. D. Šiljak, "Decentralized control and computations: status and prospects," in Annual Reviews in Control, vol. 20, 1996, pp. 131-141.

[19] S. Wang and E. Davison, "On the stabilization of decentralized control systems," in IEEE Trans. on Aut. Control, vol. 18, 1973, pp. 473-478.

[20] R. Scattolini, "Architectures for distributed and hierarchical model predictive control - a review," Journal of Process Control, vol. 19, no. 5, pp. 723-731, 2009.

[21] L. Sentis and O. Khatib, "A whole-body control framework for humanoids operating in human environments," in Proc. of the IEEE ICRA. IEEE, 2006, pp. 2641-2648.

[22] B. Siciliano and J. Slotine, "A general framework for managing multiple tasks in highly redundant robotic systems," in Int. Conf. on Adv. Rob., 1991, pp. 1211-1216.

[23] W. Shen and J. Gu, "Multi-criteria kinematics control for the pa10-7c robot arm with robust singularities," in Proc. of the IEEE Int. Conf. on Robotics \& Biomimetics, 2007, pp. 1242-1248.

[24] J. Nash, "Non-cooperative games," The Annals of Math., vol. 54, no. 2 , pp. 286-295, 1951.

[25] A. Rantzer, "Dynamic dual decomposition for distributed control," in American Control Conf., 2009. ACC'09. IEEE, 2009, pp. 884-888.

[26] S. Barthelemy, J. Salini, and A. Micaelli, "Arboris-python." [Online]. Available: https://github.com/salini/arboris-python

[27] G. Sandini, G. Metta, and D. Vernon, "The icub cognitive humanoid robot: An open-system research platform for enactive cognition," in 50 Years of Artificial Intelligence, ser. Lecture Notes in Computer Science. Springer, 2007, ch. 32, pp. 358-369. 